\title{
Distribution and niche of Suillus aurihymenius in Asia
}

\author{
Vyacheslav Vlasenko ${ }^{1 *}$, Dejidmaa Turmunkh $^{2}$ \\ ${ }^{1}$ Central Siberian Botanical Garden SB RAS, 630090 Novosibirsk, Russia \\ ${ }^{2}$ Plant Protection Research Institute of Mongolia, 17024 Ulaanbaatar, Mongolia
}

\begin{abstract}
We defined the regularities spatial distribution of Suillus aurihymenius based on the occurrence data, as well as bioclimatic characteristics in order to control species status in the natural environment. Suillus aurihymenius is a rare species known only from our collections from the Republic of Altai and Republic of Tuva. Realised niche of $S$. aurihymenius on the western border of its currently known range in larch forests in the floodplains of rivers of the steppe of southeast Altai. Conservation of natural habitats of $S$. aurihymenius in the steppes of intermontane basins in the southeast of Altai will contribute to its preservation in Russia.
\end{abstract}

\section{Introduction}

Suillus aurihymenius was first recorded by us in Russia, in the Altai Mountains in 2019 near Jazator [1]. Fruiting bodies of the fungus and habitat are shown in Fig. 1.
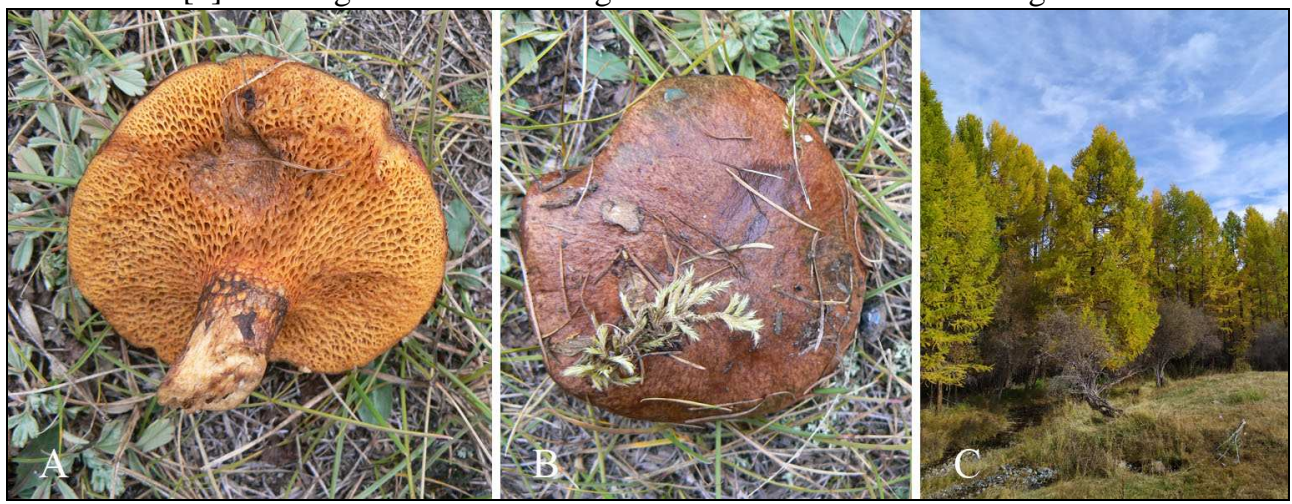

Fig. 1. A, B - Fruiting bodies of S. Aurihymenius. C - habitat of species in the Altai.

Suillus aurihymenius was described from China in 2016, from Greater Khingan Mountains, China. In China, the species grows under Larix gmelinii.

In Russia, Suillus aurihymenius growing under Larix sibirica in riverside larch forests in the mountain steppe belt in the intermountain basins of Altai Mountains.

\footnotetext{
*Corresponding author: chag_dejidmaa@yahoo.com
} 
Suillus aurihymenius is similar to $S$. tridentinus which is widespread in Europe, but differs from by its reddish gold hymenium color, stronger reddish brown discoloration of the context, and less squamulose pileus [2].

\section{Materials and methods}

\section{Field studies}

We obtained data on the distribution of the studied fungal species based on the fruiting bodies collected by us during 2019-2020 in the Republic of Altai and in 2020 in the Republic of Tuva. Voucher specimens of the studied species are stored in the MG Popov Herbarium (NSK), Novosibirsk, Russia.

Morphological examination

We performed the initial morphological examination of $S$. aurihymenius fruiting bodies using Carl Zeiss Axioskop-40 light microscope.

Molecular genetic studies

The ITS1-5.8S-ITS2 region nrDNA of $S$. aurihymenius has been sequenced for sample NSK 1014458, collected near Jazator, GenBank access number MT302579.

Biodiversity data overview and Study area

We obtained the three locations for species from the publications [3]. In total, we included six locations of $S$. aurihymenius in Asia, including our three locations (all points do not do not fall into one raster cell). Distribution of S. aurihymenius in Asia and locations used in the niche modeling analysis shows in Fig 2.

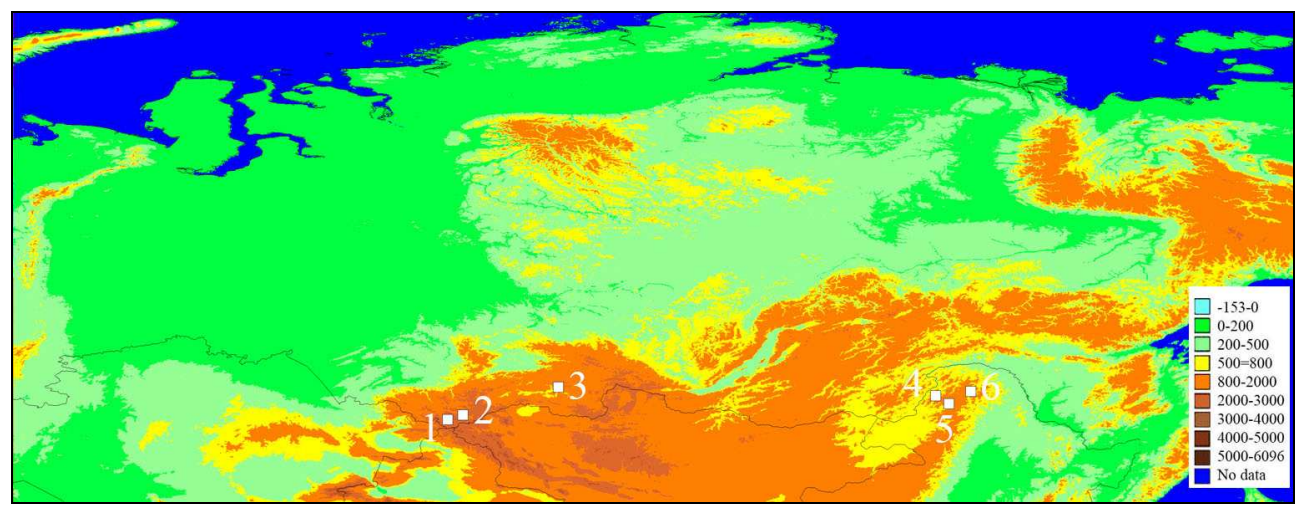

Fig. 2. Locations of S. aurihymenius in Asia. Russia: Altay Mts., Republic of Altai, Kosh-Agachsky district, Loc. 1. - near Jazator (Belyashi), $49.6914^{\circ} \mathrm{N}, 87.4344^{\circ} \mathrm{E}, 1569 \mathrm{~m}$, NSK 1014458; Loc. 2. near Ortolyk, 50.0190 N, 88.4785 E, 1764 m, NSK 1014685, 1014686, 1014688, 1014689; Sayan Mts., Republic of Tuva, Piy-Khemsky District, Tapsinsky Wildlife Sanctuary, Loc. 3. - near Seserlig, 51.9135 ${ }^{\circ} \mathrm{N}, 94.9804^{\circ} \mathrm{E}, 1551 \mathrm{~m}$, NSK 1014605. China: Greater Khingan Mts., Heilongjiang, Huzhong Nature Reserve, 51.6166 ${ }^{\circ} \mathrm{N}, 123.0502^{\circ} \mathrm{E}, 800 \mathrm{~m}$, HKAS 63129; Inner Mongolia: near Genhe, approx. 50.7759 $\mathrm{N}, 121.5590^{\circ} \mathrm{E}, 713 \mathrm{~m}$, HKAS 63130; Argun River Area, Mordaga Native Forest Park, $51.3166^{\circ} \mathrm{N}, 120.6666^{\circ} \mathrm{E}, 540 \mathrm{~m}$, HKAS 63131. The colors corresponds to the height above sea level (decoding in the legend).

\section{SDM algorithms, predictor type, software and data used}

We used the computer program DIVA-GIS [4] for mapping and geographic data analysis (https://www.diva-gis.org/). We downloaded data on the relief and the heights of the studied locations, as well as global data on current climat ( 1950-2000) from https://www.diva-gis.org/Data and https://www.diva-gis.org/climate, source Worldclim, version 1.3. All the 19 environmental layers of Bioclim, BIO1-BIO19 [5] constructed using 
the DIVA-GIS with 2.5-minute resolution (30 arc seconds square) encompassed most of the Asia.

We constructed the climatic profile of $S$. aurihymenius in Asia using the BIOCLIM method. This method developed by $\mathrm{H}$. Nix [5] constructs histograms of bioclimatic variables that reflect species climate profile. The DIVA-GIS software implements the BIOCLIM method.

\section{Results and Discussion}

The climatic niche of a species bases on the identification of the climatic characteristics of the locations where the studied species appeared [6].

We generated the frequency histograms based on bioclimatic variables BIO1 (Annual Mean Temperature) and BIO12 (Annual Precipitation) (Fig 3). The histograms show the distribution of $S$. aurihymenius along the customized ranges for selected climatic variables.

We carried out the modeling of a two-dimensional niche for S. aurihymenius based on bioclimatic variables BIO1 (Annual Mean Temperature) and BIO12 (Annual Precipitation) (Fig 4).

We narrowed the climatic niche in order to determine the core of the climatic niche with the most favorable habitat parameters for the species, based on the selected bioclimatic variables.

We adjusted the width of the niche by changing the percentile values. We changed the boundaries of the two-dimensional niche by changing the percentile to the limit when the points went outside the range values for 19 bioclimatic variables (in the model, the points of presence changed color to red).

Next, we narrowed the niche until all points went beyond it (limit $=0.401)$. In this way we changed the limits of the two-dimensional niche within $0.000-0.001-0.201-0.400 \geq$ $0.401(100 \%-50 \%-16.7 \%-16.7 \%-0 \%$ observations presented in a niche).
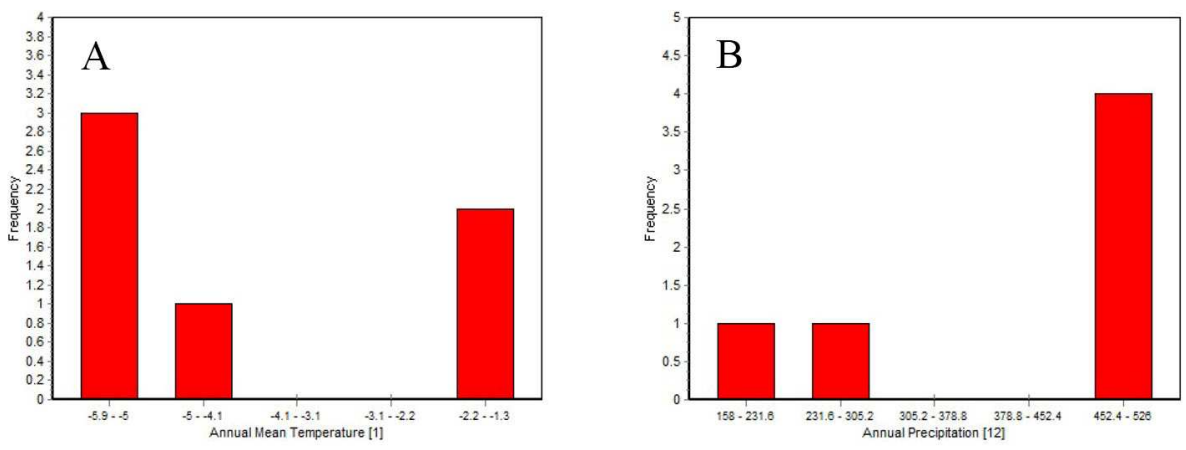

Fig. 3. Frequency histograms, which show the distribution of $S$. aurihymenius along customized ranges. A - Annual Mean Temperature (BIO1), B - Annual Precipitation (BIO12). Axis of abscissa the factor strength. Ordinate axis - the frequencies. 


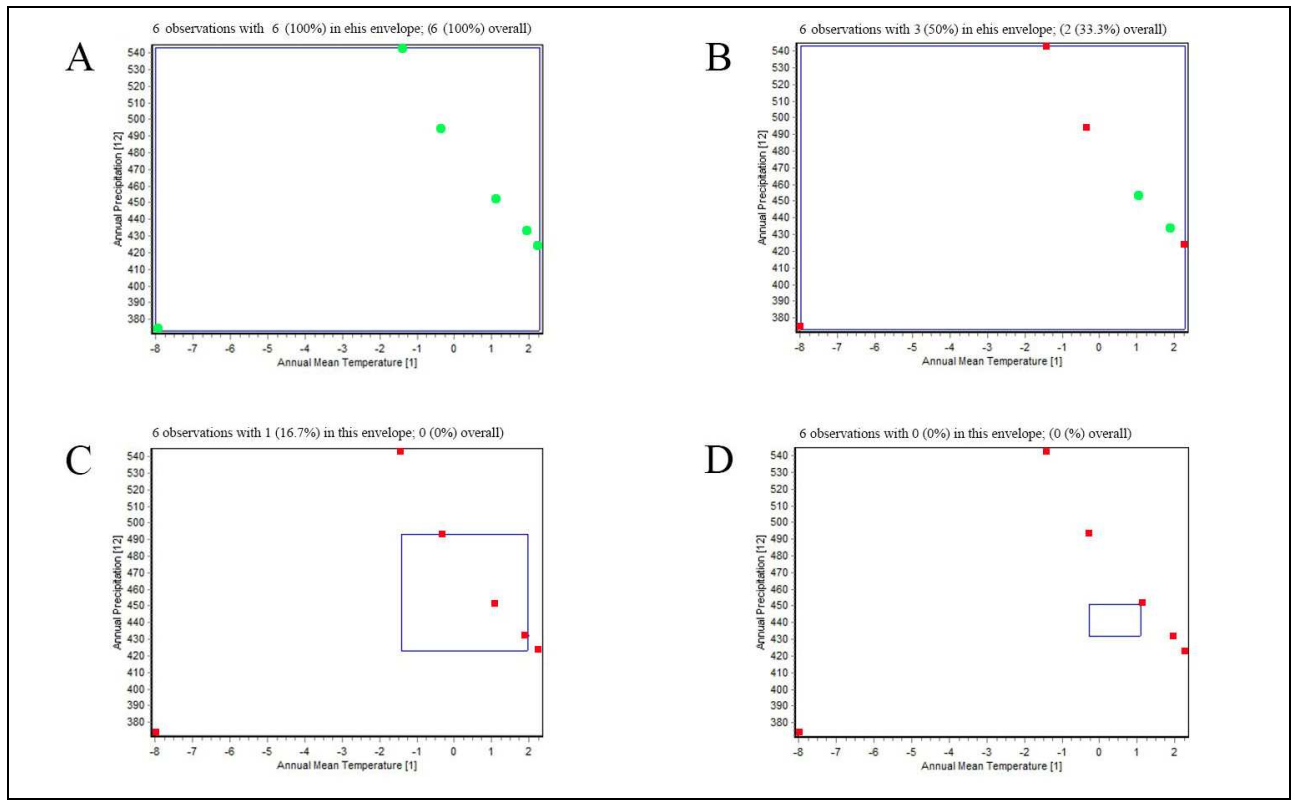

Fig. 4. Visualization of a two-dimensional niche for $S$. aurihymenius based on two climatic variables using the Envelope method. Limits of the two-dimensional niche (0.000-0.400): A - 0.000, B - 0.001, $\mathrm{C}-0.201, \mathrm{D}-0.401$. Blue rectangle is the climatic niche. The green points represent the presence points with a climate profile within the range limits of all the 19 Bioclim climatic variables. Red square within the blue rectangle represent presence points with a climate profile within the values of the range limits for the selected variables (Annual Mean Temperature and Annual Precipitation), but with one or more values laying outside the range limits of the other 17 Bioclim variables. Red square outside the blue rectangles represent presence points with a climate profile with one or more values laying outside the range limits of the 19 Bioclim climatic variables.

We narrowed the climatic niche in order to determine the core of the climatic niche with the most favorable habitat parameters for the species, based on the selected bioclimatic variables (Fig 5).

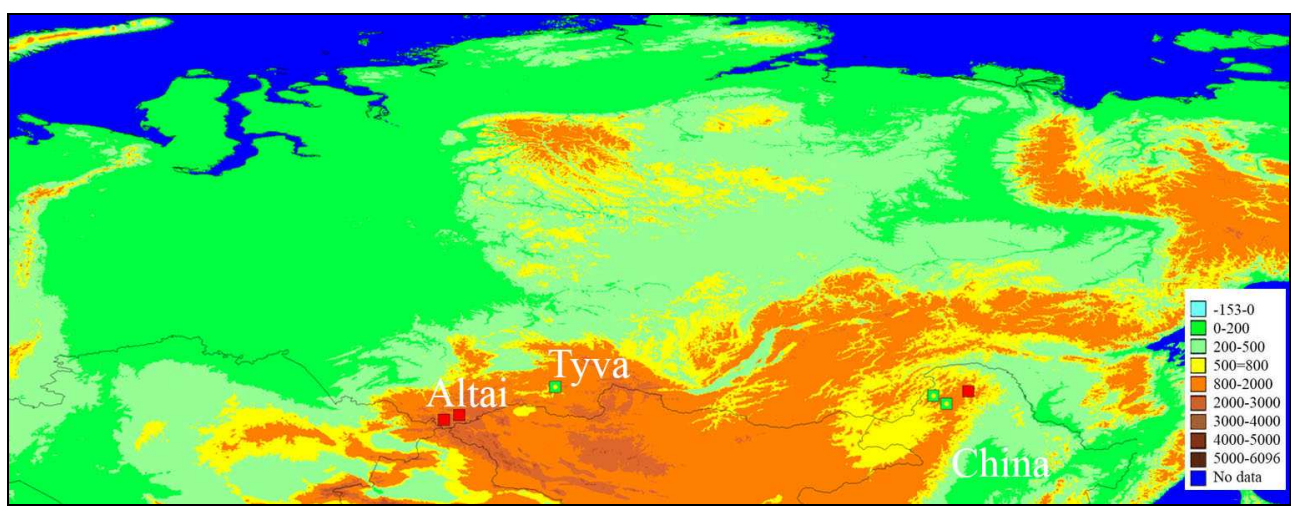

Fig. 5. The environment core of the species range (green points) of $S$. aurihymenius in Asia with the two-dimensional niche limits $=0.201$.

\section{Conclusions}


The constructed niche model based on the selected bioclimatic variables showed that $S$. aurihymenius on the border of its currently known range - on the western border in larch forests in the floodplains of rivers of the steppe of southeast Altai, as well as in the extreme eastern point in China, is located on the border of the bioclimatic niche with the least favorable conditions.

Larch forests in the steppe are located in an area with a very harsh climate in the Chuya steppe - an intermontane basin in the southeast of Altai. Larch forests are able to exist here only thanks to the rivers in the floodplains of which they grow.

Since the species of the genus Suillus are mycorrhizal fungi, their distribution is directly related to the distribution of a tree species - a symbiont. Therefore, conservation of larch forests in steppe of intermontane basins in the southeast of Altai will contribute to conservation of $S$. aurihymenius in Russia.

Acknowledgements. The work was funded by RFBR and MCESSM according to the research project 19-54-44002 Mong_T.

\section{References}

1. S. Yu. Bolshakov, L. B. Kalinina, S. V. Volobuev, Yu. A. Rebriev, A. G. Shiryaev, Yu. R. Khimich, V. A. Vlasenko, A. V. Leostrin, N. V. Shakhova, A. V. Vlasenko, T. Dejidmaa, O. N. Ezhov, I. V. Zmitrovich, Mikol. I fitopatol., 54, 6 (2020)

2. X.-F. Shi, F.-Q. Yu, R. Zhang, P.-G. Liu, Mycotaxon, 131 (2016)

3. R. J. Hijmans, L. Guarino, P. Mathur. DIVA-GIS Version 7.5 Manual. Available from http://diva-gis.org/docs/DIVA-GIS_manual_7.pdf (2012)

4. R. J. Hijmans, S. E. Cameron, J. L. Parra, P. G. Jones, A. Jarvis. Int. J. Climatol., 25 (2005)

5. H. A. Nix, Australian flora and fauna. Series 7. Atlas of elapid snakes of Australia (Australian Government Publishing Service, Canberra, 1986)

6. X. Scheldeman, M. Van Zonneveld, Training manual on spatial analysis of plant diversity and distribution (Biodiversity International, Rome, 2010) 